\title{
Erratum to: String and M-Theory: Answering the Critics
}

\section{M.J. Duff}

Published online: 22 December 2012

(C) Springer Science+Business Media New York 2012

\section{Erratum to: Found Phys (2013) 43:182-200 DOI 10.1007/s10701-011-9618-4}

Following are corrections to the original article:

Page numbers in reference to "The Trouble with Physics" refer to the version the author was sent to review by Nature Physics and may be different in published versions.

The quote in Section 2.1, page 187, "It does not take much foresight or courage to think about strings" should read: "Nor does it take much foresight or courage to think about these things when hundreds of other people are thinking the same thoughts."

Page 190, Section 3, Schneider should read Snyder.

Page 192, last line, cachophany should read cacophony.

Corrected Ref. [5] is Distler, J., Garibaldi, S.: There is no "theory of everything" inside E8. Commun. Math. Phys. 298:419-436 (2010).

The online version of the original article can be found under doi:10.1007/s10701-011-9618-4.

M.J. Duff $(\bowtie)$

The Blackett Laboratory, Imperial College London, Prince Consort Road, London SW7 2AZ, UK

e-mail: m.duff@imperial.ac.uk 